\title{
Two-year clinical outcomes in stable angina and acute coronary syndrome after percutaneous coronary intervention of left main coronary artery disease
}

Dae Young Hyun, Myung Ho Jeong, Doo Sun Sim, Yun Ah Jeong, Kyung Hoon Cho, Min Chul Kim, Hyun Kuk Kim, Hae Chang Jeong, Keun Ho Park, Young Joon Hong, Jun Han Kim, Youngkeun Ahn, and Jung Chaee Kang

The Heart Center of Chonnam National University Hospital and The Heart Research Center Nominated by Korea Ministry of Health and Welfare, Gwangju, Korea

\section{Korean J Intern Med 2016;31:1084-1092 \\ https://doi.org/10.3904/kjim.2014.373}

In the article cited above, fourth line of the first paragraph in Methods part, page 1085, has an error. Chonbuk National University Hospital should be corrected as "Chonnam National University Hospital."

We apologize for any inconvenience that this may have caused. 